\title{
Effects of Various Antibiotics Alone or in Combination with Doripenem against Klebsiella pneumoniae Strains Isolated in an Intensive Care Unit
}

\author{
Berna Ozbek Celik, ${ }^{1}$ Emel Mataraci-Kara, ${ }^{1}$ and Mesut Yilmaz ${ }^{2}$ \\ ${ }^{1}$ Department of Pharmaceutical Microbiology, Faculty of Pharmacy, Istanbul University, Beyazit, 34116 Istanbul, Turkey \\ ${ }^{2}$ Department of Infectious Diseases and Clinical Microbiology, Faculty of Medicine, University of Istanbul Medipol, \\ 34083 Istanbul, Turkey \\ Correspondence should be addressed to Berna Ozbek Celik; bernaozbek@hotmail.com
}

Received 4 July 2014; Accepted 1 September 2014; Published 28 October 2014

Academic Editor: Abdelwahab Omri

Copyright (C) 2014 Berna Ozbek Celik et al. This is an open access article distributed under the Creative Commons Attribution License, which permits unrestricted use, distribution, and reproduction in any medium, provided the original work is properly cited.

\begin{abstract}
Colistin, tigecycline, levofloxacin, tobramycin, and rifampin alone and in combination with doripenem were investigated for their in vitro activities and postantibiotic effects (PAEs) on Klebsiella pneumoniae. The in vitro activities of tested antibiotics in combination with doripenem were determined using a microbroth checkerboard technique. To determine the PAEs, K. pneumoniae strains in the logarithmic phase of growth were exposed for $1 \mathrm{~h}$ to antibiotics, alone and in combination. Recovery periods of test cultures were evaluated using viable counting after centrifugation. Colistin, tobramycin, and levofloxacin produced strong PAEs ranging from 2.71 to $4.23 \mathrm{~h}$, from 1.31 to $3.82 \mathrm{~h}$, and from 1.35 to 4.72 , respectively, in a concentration-dependent manner. Tigecycline and rifampin displayed modest PAEs ranging from $1.18 \mathrm{~h}$ to $1.55 \mathrm{~h}$ and 0.92 to 1.19 , respectively. Because it is a beta-lactam, PAEs were not exactly induced by doripenem (ranging from 0.10 to $0.18 \mathrm{~h}$ ). In combination, doripenem scarcely changed the duration of PAE of each tested antibiotic alone. The findings of this study may have important implications for the timing of doses during K. pneumoniae therapy with tested antibiotics.
\end{abstract}

\section{Introduction}

Klebsiella pneumoniae can cause different types of healthcareassociated infections, including pneumonia, bloodstream infections, wound or surgical site infections, and meningitis [1]. In healthcare settings, patients whose care requires devices like ventilators or intravenous catheters and patients taking long courses of certain antibiotics are most at risk for Klebsiella infections $[1,2]$. Because K. pneumoniae has the ability to acquire resistance against different classes of antibiotics, including carbapenems [3], it has been an important consideration in the development of effective combination therapy, both to rapidly enhance bactericidal activity and to help prevent or delay the emergence of resistance $[4,5]$. Studies have proven that combinations of a doripenem with colistin or tigecycline or tobramycin or levofloxacin or rifampin can produce synergy [6-11].
Additionally, optimization of dosing by pharmacodynamic parameters has also been shown to improve outcomes of $K$. pneumoniae infections $[12,13]$.

PAE, a pharmacodynamic parameter, is defined as the suppression of bacterial growth observed after removal of an antimicrobial agent from the culture medium [14-16]. Additionally, extending the dosing interval of an antimicrobial agent with a PAE has potential advantages: for example, reduced cost and less toxicity [17]. PAE can be used to develop more effective dosing regimens to improve the efficiency of antimicrobial agents, reduce the emergence of resistance, and develop new drugs and new formulations and should be considered during guideline formation and development [18].

According to our research, a limited number of reports addressing the PAE of antibiotics have been published regarding $K$. pneumonia. Therefore, the present study aimed to identify the PAE interaction of the different groups of 
antibiotics alone or in combination with doripenem against K. pneumoniae strains isolated from bloodstream infections. Furthermore, we investigated whether PAEs induced by the tested antibiotic combinations differ from those induced by colistin, tigecycline, levofloxacin, tobramycin, or rifampicin alone for K. pneumoniae strains.

\section{Materials and Methods}

2.1. Bacterial Isolates. Six nonduplicate, nosocomially acquired $K$. pneumoniae strains isolated from blood specimens between January and June 2011 were obtained from the Department of Infectious Diseases and Clinical Microbiology, Faculty of Cerrahpasa Medicine, Istanbul University. All strains were identified using API 20NE (bioMérieux). As a reference strain, K. pneumonia ATCC 700603 (American Type Culture Collection, Rockville, MD, USA) was used throughout the study to verify the accuracy of microdilution test procedure to ensure that MIC values of the antibiotics studied were within the accuracy range stated by the Clinical and Laboratory Standards Institute (CLSI) [19].

2.2. Antibiotics. All antimicrobial agents were kindly provided by their respective manufacturers. Stock solutions of colistin sulphate, tobramycin, levofloxacin, and rifampin were stored frozen at $-80^{\circ} \mathrm{C}$. Frozen solutions of antibiotics were used within six months. Tigecycline and doripenem solutions were prepared on the day of use.

2.3. Media. Mueller-Hinton broth (Difco Laboratories, Detroit, MI, USA) was used for MIC, and PAE studies, and supplemented with $25 \mathrm{mg}$ of calcium/liter and $12.5 \mathrm{mg}$ of magnesium/liter (CAMHB). The broth was used within $24 \mathrm{~h}$ of preparation for the tigecycline [20]. Pour plates of tryptic soy agar (Difco Laboratories) were used for colony counts.

2.4. MIC Determinations. MICs were determined by the microbroth dilution technique described by CLSI. Serial twofold dilutions ranging from 512 to $0.250 \mathrm{mg} / \mathrm{L}$ for rifampin, from 128 to $0.06 \mathrm{mg} / \mathrm{L}$ for doripenem, tobramycin, levofloxacin, and from 32 to $0.015 \mathrm{mg} / \mathrm{L}$ for tigecycline and colistin were prepared in fresh CSMHB 96-well microtiter plates. The inoculum was prepared with a 4 - to 6 -h broth culture. Each isolate was adjusted spectrophotometrically to $1 \times 10^{8} \mathrm{CFU} / \mathrm{mL}\left(\mathrm{OD}_{600} 0.12-0.13\right)$ and diluted in CSMHB to create a final concentration of $5 \times 10^{5} \mathrm{CFU} / \mathrm{mL}$ in the test tray. The trays were covered, placed in plastic bags to prevent evaporation, and incubated at $37^{\circ} \mathrm{C}$ for $18-20 \mathrm{~h}$. The MIC was defined as the lowest concentration of antibiotic giving complete inhibition of visible growth.

\subsection{Determination of the Fractional Inhibitory Concentra-} tion Index. The effects of antibiotics in combination were assessed using a microbroth checkerboard technique [21]. Each microtiter well containing the mixture of antibiotics was inoculated with a 4 - to 6-hour broth culture diluted to produce a final concentration of approximately $5 \times 10^{5} \mathrm{CFU} / \mathrm{mL}$. After incubation at $37^{\circ} \mathrm{C}$ for $18-20 \mathrm{~h}$, the fractional inhibitory concentration (FIC) index was determined as the combined concentration divided by the single concentration. The combination value was derived from the highest dilution of antibiotic combination permitting no visible growth. With this method, synergy was defined as an FIC index $\leq 0.5$, no interaction as an FIC index between 0.5 and 4, and antagonism as an FIC index $\geq 4.0$ [22].

2.6. Determination of PAE. PAEs were determined by a standard viable counting method [15]. Samples were incubated for $1 \mathrm{~h}$ to avoid prolonged antibiotic exposure and consequent complete eradication of the organism. At time zero, $1 \mathrm{~mL}$ of inoculum was added to tubes containing $29 \mathrm{~mL}$ CSMHB with or without test antibiotics. Organisms in the logarithmic phase of growth, producing a final concentration of inoculums in the test tubes of approximately $10^{6} \mathrm{CFU} / \mathrm{mL}$, were exposed to concentrations of doripenem, tigecycline, colistin, levofloxacin, tobramycin, and rifampin equal to $1 \mathrm{x}$ MIC or $4 \mathrm{x}$ MIC, alone or in combination. After incubation for $1 \mathrm{~h}$ in a $37^{\circ} \mathrm{C}$ shaking water bath, antibiotics were removed by centrifugation at 5,000 rpm for $10 \mathrm{~min}$. Then supernatant was decanted and cells were washed twice in buffered sterile saline $(0.9 \% \mathrm{NaCl})$ before being resuspendedin $30 \mathrm{~mL}$ of prewarmed CSMHB. Bacterial counts of tube contents were determined at time zero, immediately before and after centrifugation, and each $\mathrm{h}$ after centrifugation for $8 \mathrm{~h}$ by spreading on pour platesusing 10 -fold dilutions in cold saline as required. Antimicrobial carryover was controlled by the inhibition of colonial growth at the site of the initial streak according to NCCLS guidelines [23].

Plates were read after incubation for $18-24 \mathrm{~h}$ at $37^{\circ} \mathrm{C}$. The PAE was defined according to Craig and Gudmundsson [Craig] as PAE $=T-C$, where $T$ is the time (in $h$ ) required for the viability count in the test culture to increase $1 \log _{10}$ above the count observed immediately after centrifugation, and $C$ is the corresponding time for the controls. Experiments were conducted in triplicate.

2.7. Statistical Analysis. Statistical analysis was done with GraphPad Prism 5.0 (GraphPad Software Inc., San Diego, CA, USA). Results are expressed as mean \pm SD. One-way ANOVA followed by Bonferroni's multiple comparison test was performed to examine the change in PAE values of each antibiotic concentration alone and in combination. In the results, alpha $<0.05$ was considered significant.

\section{Results}

The MICs of doripenem, colistin, tobramycin, levofloxacin, tigecycline, and rifampin against six tested clinical strains and the reference strains of K. pneumoniae ATCC 700603 are shown in Table 1 . With an FIC index $\leq 0.5$ as the borderline value, synergy was detected against 1 strain with the doripenem-tigecycline and the doripenem-colistin combination, and against 2 strains with the doripenem-levofloxacin and doripenem-tobramycin combinations (Table 1). Antagonism was not observed with any combination. 
TABLE 1: In vitro activities of antibiotics alone (MIC, $\mathrm{mg} / \mathrm{L}$ ) and in combination (FIC index) with doripenem against the studied strains.

\begin{tabular}{|c|c|c|c|c|c|c|c|}
\hline & KP-700606 & KP-1 & KP-2 & KP-3 & KP-4 & KP-5 & KP-6 \\
\hline \multicolumn{8}{|l|}{ Antibiotic } \\
\hline DOR & 0.06 & 16 & 16 & 16 & 16 & 16 & 16 \\
\hline CS & 1 & 0.5 & 0.25 & 1 & 1 & 1 & 1 \\
\hline TGC & 0.12 & 0.25 & 0.125 & 0.125 & 0.06 & 0.25 & 0.125 \\
\hline TOB & 0.5 & 2 & 1 & 2 & 1 & 0.5 & 1 \\
\hline LVX & 0.06 & 1 & 0.03 & 0.06 & 0.125 & 0.5 & 2 \\
\hline RIF & 512 & 256 & 256 & 512 & 256 & 512 & 512 \\
\hline \multicolumn{8}{|l|}{ Combination } \\
\hline $\mathrm{DOR}+\mathrm{TGC}$ & 0.75 & 1 & 0.75 & 0.75 & 0.25 & 1 & 0.75 \\
\hline $\mathrm{DOR}+\mathrm{CS}$ & 0.75 & 1 & 0.5 & 1 & 0.75 & 1 & 0.75 \\
\hline $\mathrm{DOR}+\mathrm{LVX}$ & 0.5 & 1 & 0.5 & 1 & 0.125 & 0.75 & 1 \\
\hline $\mathrm{DOR}+\mathrm{TOB}$ & 1 & 0.25 & 1 & 1 & 0.25 & 1 & 1 \\
\hline $\mathrm{DOR}+\mathrm{RIF}$ & 1 & 1 & 0.75 & 1 & 2 & 1 & 0.75 \\
\hline
\end{tabular}

DOR: doripenem; TGC: tigecycline; CS: colistin sulphate; LVX: levofloxacin; TOB: tobramycin; RIF: rifampin. KP-700606: reference strain (Klebsiella pneumoniae ATCC 700606); KP: Klebsiella pneumoniae.

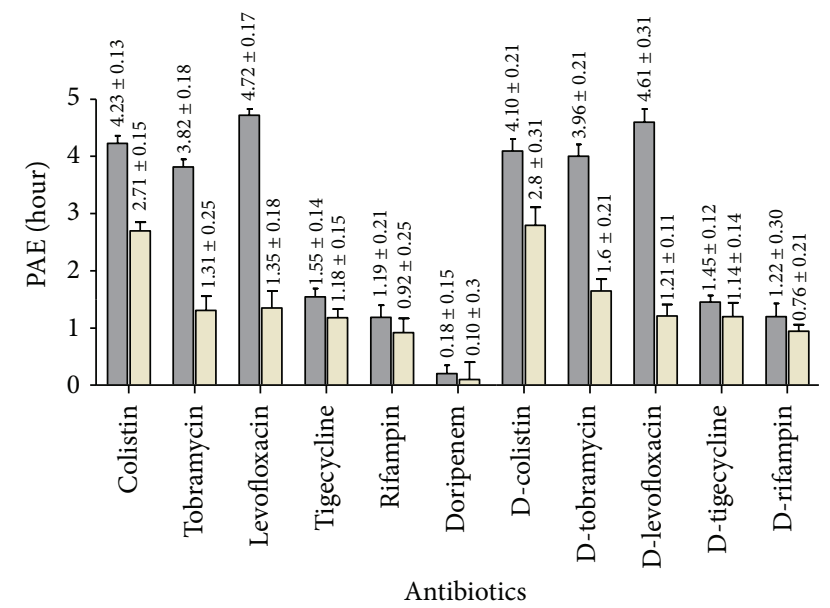

$\square$ 4x MIC

Figure 1: The mean PAE values for six clinical strains of $K$. pneumonia.

The mean PAE values for six clinical strains of $K$. pneumoniae are displayed in Figure 1. For a concentration of 1x MIC, colistin, tobramycin, and levofloxacin showed a good PAE for all strains, varying between 2.34 and $3.13 \mathrm{~h}$, between 1.01 and $1.59 \mathrm{~h}$, and between 1.05 to $1.56 \mathrm{~h}$, respectively. Tigecycline and rifampin showed a modest PAE for all strains, varying between 0.95 and $1.39 \mathrm{~h}$ and between 0.73 and $1.37 \mathrm{~h}$, respectively. When the concentrations of tested antibiotics were increased to $4 \mathrm{x}$ MIC, the duration of the PAEs were significantly prolonged: colistin produced PAEs ranging from 3.92 to $4.51 \mathrm{~h}(P<0.05)$; levofloxacin produced PAEs ranging from 4.42 to $4.92 \mathrm{~h}(P<0.0001)$; and tobramycin produced PAEs ranging from 3.56 to $4.16 \mathrm{~h}(P<0.001)$. Although tigecycline and rifampin at $4 \mathrm{x}$ MIC produced PAEs from 1.38 to 1.77 and from 0.92 to 1.45 , respectively, in a concentrationdependent manner, no statistically significant difference was found $(P>0.05)$. On the other hand, negligible PAE values were obtained with doripenem at both $1 \mathrm{x}$ MIC or $4 \mathrm{x}$ MIC against the studied strains. As seen in Figure 1, doripenem combined with the tested antibiotics at concentrations of $1 \mathrm{x}$ MIC or $4 \mathrm{x}$ MIC did not produce significantly different PAEs than when the antibiotics were used alone $(P>0.05)$.

\section{Discussion}

Determination of the postantibiotic effects is an important part of preclinical evaluation of antibiotics because it is a factor that influences antibiotic dosing intervals [36-38]. PAE is likely the result of several mechanisms, including nonlethal damage caused by the antibiotic and continued persistence of the drug at the bacterial drug-binding site for a given period of time after the drug is removed [39]. For example, recovery from the postantibiotic effect induced by tobramycin in Escherichia coli depends upon reestablishment of protein synthesis, and recovery from the levofloxacininduced postantibiotic effect depends upon restoration of DNA synthesis [33].

In the present study, PAEs of all tested antibiotics were determined and compared with the previous studies in Table 2. Fluoroquinolones generally produce PAEs against Gram-negative and positive strains [24, 25, 29, 40, 41]. Consistent with previous study by Spangler et al. [24] our results display that levofloxacin possesses strong PAE values against $K$. pneumoniae strains. This antibiotic at $4 \mathrm{x}$ MIC concentrations alone exhibited the most prolonged PAEs compared to all tested antibiotics. Since the clinical implication of long PAEs lies in the possibility of increasing the intervals between drug administrations, thus allowing for fewer daily dosages and thereby potentially reducing treatment costs, increasing patient compliance and decreasing drug exposure [42], administering once-daily levofloxacin might be advantageous for patient outcomes. 
TABLE 2: The PAE values for tested antibiotics.

\begin{tabular}{|c|c|c|c|c|c|}
\hline Antibiotic & Bacteria & Special conditions & $\begin{array}{c}\text { Duration of PAE } \\
(\mathrm{H})\end{array}$ & Author/year & $\begin{array}{c}\text { Reference } \\
\text { number }\end{array}$ \\
\hline \multirow{2}{*}{ Levofloxacin } & K. pneumoniae & 1 and $4 \mathrm{x}$ MIC & 1.35 and 4.72 & Present study & \\
\hline & K. pneumoniae & $0.5 \mathrm{x} \mathrm{MIC}$ & 1.80 & Spangler et al./2000 & {$[24]$} \\
\hline \multirow{5}{*}{ Colistin } & K. pneumoniae & 1 and $4 \mathrm{x}$ MIC & 2.71 and 4.23 & Present study & \\
\hline & A. baumannii & 1 and $4 \mathrm{x}$ MIC & 3.00 and 6.28 & Özbek and Şentürk/2010 & {$[25]$} \\
\hline & P. aeruginosa & 1 and $20 x$ MIC & 1.13 and 2.12 & Bozkurt-Güzel and Gerçeker/2012 & {$[26]$} \\
\hline & P. aeruginosa & 16x MIC & 2.00 and 3.00 & Li et al./2001 & {$[27]$} \\
\hline & $\begin{array}{l}\text { A. baumannii } \\
\text { ATCC } 19606\end{array}$ & 16,32 and $64 \mathrm{x}$ MIC & $1.00,2.30$ and 3.50 & Owen et al./2007 & {$[28]$} \\
\hline \multirow{4}{*}{ Tobramycin } & K. pneumoniae & 1 and $4 \mathrm{x}$ MIC & 1.31 and 3.82 & Present study & \\
\hline & P. aeruginosa & 1 and $10 x$ MIC & 1.50 and 3.10 & Ozbek and Otuk/2009 & {$[29]$} \\
\hline & P. aeruginosa & In vivo & 2.00 to 4.00 & Gudmundsson et al./1993 & {$[30]$} \\
\hline & $\begin{array}{c}\text { Gram-negative } \\
\text { bacteria }\end{array}$ & In vivo & 3.00 to 4.00 & Spivey/1992 & {$[17]$} \\
\hline \multirow{3}{*}{ Tigecycline } & K. pneumoniae & 1 and $4 \mathrm{x}$ MIC & 1.18 and 1.55 & Present study & \\
\hline & K. pneumoniae & 2 and 10x MIC & 1.70 and 1.80 & Pankuch and Appelbaum/2009 & {$[31]$} \\
\hline & $\begin{array}{c}\text { Enterococcus } \\
\text { faecalis }\end{array}$ & 1 to $20 \mathrm{x}$ MIC & 1.00 and 4.50 & Lefort et al./2003 & {$[32]$} \\
\hline \multirow{3}{*}{ Rifampin } & K. pneumoniae & 1 and $4 \mathrm{x}$ MIC & 0.92 and 1.19 & Present study & \\
\hline & E. coli ATCC 25922 & 5x MIC & 4.00 & Stubbings et al./2006 & {$[33]$} \\
\hline & $\begin{array}{c}\text { Legionella } \\
\text { pneumophila }\end{array}$ & 4x MIC & 2.86 and 3.09 & Dubois and St-Pierre/2000 & {$[34]$} \\
\hline \multirow[b]{2}{*}{ Doripenem } & K. pneumoniae & 1 and $4 \mathrm{x}$ MIC & 0.10 and 0.18 & Present study & \\
\hline & $\begin{array}{c}\text { E. coli and } P \text {. } \\
\text { aeruginosa }\end{array}$ & 10x MIC & $\begin{array}{l}\text { Weak or No PAE } \\
\text { for meropenem }\end{array}$ & Odenholt-Tornqvist/1993 & {$[35]$} \\
\hline
\end{tabular}

The results of this research also indicate that colistin has potent PAEs against the tested $K$. pneumoniae strains. Similar to a previous study [25-28], in the present study this antibiotic displayed powerful PAEs: at $4 \mathrm{x}$ MIC alone, it exhibited nearly twice as long PAEs than when the antibiotic was used at $1 \mathrm{x}$ MIC against the tested strains. However, a recent increase in the prevalence of multidrug resistant $K$. pneumoniae and the lack of novel agents in development calls for a need to reexamine the colistin therapy.

On the other hand, the present investigation showed that tobramycin has significant PAEs against the tested six $K$. pneumonia strains. This is in agreement with the previous results $[17,29,30]$. This antibiotic at $4 \mathrm{x}$ MIC alone prolonged PAEs by more than three times than when the antibiotic was used at $1 \mathrm{x}$ MIC concentrations. The benefit of this prolonged PAE value of tobramycin may allow for prolonged dose intervals without reduced efficacy and possibly a lower frequency of adverse events during K. pneumoniae therapy.

A limited number of reports addressing tigecycline PAE have been published focusing on K. pneumoniae [31, 32]. All these studies and ours have shown that the activity of tigecycline's PAE, which has also been evaluated both in vitro and in vivo, is good and changes with increasing concentrations.

Inter alia, antibiotic combinations which include rifampin may have a role in the treatment of $K$. pneumoniae and possibly slow the selection of heteroresistant subpopulations during therapy $[11,43]$. According to PAE studies on rifampin, this antibiotic induced a postantibiotic effect against E. coli [33] and Legionella spp. [34]. So far, ours is the first study in which a PAE for rifampin has been clearly demonstrated on $K$. pneumoniae. In the present study, a moderate PAE was produced by rifampin both at $1 \mathrm{x}$ MIC and at $4 \mathrm{x}$ MIC against the studied strains.

Lastly, very negligible PAE was produced by doripenem at 1x MIC or 4x MIC against the studied strains. Although there is no prior publication on the PAEs of doripenem against $K$. pneumoniae, our results were aligned with similar results of previous studies suggesting that PAEs have been described for wide variety of antibiotics used singly against Gram-negative strains, but only for non-beta-lactams, with the exception of carbapenems on $P$. aeruginosa $[35,44,45]$.

The increasing interest in combination therapy for $K$. pneumoniae infections is mostly due to the organism's ability to acquire resistance against different classes of antibiotics, including carbapenems, with a limited availability of effective agents [3]. In our in vitro study, synergistic activity for each combination was seen at least against one clinical strain, except for doripenem-rifampin.

The other purpose of this study was to examine whether PAEs induced by drug combinations differed from PAEs induced by the drugs alone. The tested combinations produced similar PAEs from the PAEs induced by the colistin or tobramycin or levofloxacin or tigecycline or rifampin alone; statistically significant differences in PAEs were not determined, comparatively $(P>0.05)$.

Consequently, the main findings of this study are that PAE values of tested antibiotics (except for doripenem), levofloxacin, colistin, tobramycin, tigecycline, and rifampicin, 
have an ability to produce PAEs against $K$. pneumoniae and may have important implications for the dosing regimen treatment of K. pneumoniae infections. Also, PAEs induced by drug combinations were not different from PAEs induced by the drugs alone.

\section{Conflict of Interests}

The authors declare that there is no conflict of interests regarding the publication of this paper.

\section{Acknowledgment}

This work was supported by a Grant from the Research Fund of The University of Istanbul, Project no. 4703.

\section{References}

[1] R. Podschun and U. Ullmann, "Klebsiella spp. as nosocomial pathogens: epidemiology, taxonomy, typing methods, and pathogenicity factors," Clinical Microbiology Reviews, vol. 11, no. 4, pp. 589-603, 1998.

[2] A. Asensio, A. Oliver, P. González-Diego et al., "Outbreak of a multiresistant Klebsiella pneumoniae strain in an intensive care unit: antibiotic use as risk factor for colonization and infection," Clinical Infectious Diseases, vol. 30, no. 1, pp. 55-60, 2000.

[3] M. Akova, G. L. Daikos, L. Tzouvelekis, and Y. Carmeli, "Interventional strategies and current clinical experience with carbapenemase-producing Gram-negative bacteria," Clinical Microbiology and Infection, vol. 18, no. 5, pp. 439-448, 2012.

[4] Z. Z. Deris, H. H. Yu, K. Davis et al., "The combination of colistin and doripenem is synergistic against Klebsiella pneumoniae at multiple inocula and suppresses colistin resistance in an in vitro pharmacokinetic/pharmacodynamic model," Antimicrobial Agents and Chemotherapy, vol. 56, pp. 5103-5112, 2012.

[5] J. A. Korvick, C. S. Bryan, B. Farber et al., "Prospective observational study of Klebsiella bacteremia in 230 patients: outcome for antibiotic combinations versus monotherapy," Antimicrobial Agents and Chemotherapy, vol. 36, no. 12, pp. 2639-2644, 1992.

[6] A. Elemam, J. Rahimian, and M. Doymaz, "In vitro evaluation of antibiotic synergy for polymyxin B-resistant carbapenemaseproducing Klebsiella pneumoniae," Journal of Clinical Microbiology, vol. 48, no. 10, pp. 3558-3562, 2010.

[7] M. G. Jernigan, E. G. Press, M. H. Nguyen, C. J. Clancy, and R. K. Shields, "The combination of doripenem and colistin is bactericidal and synergistic against colistin-resistant, carbapenemaseproducing Klebsiella pneumoniae," Antimicrobial Agents and Chemotherapy, vol. 56, no. 6, pp. 3395-3398, 2012.

[8] B. Kadar, B. Kocsis, A. Toth, and et al, "Synergistic antibiotic combinations for colistin-resistant Klebsiella pneumoniae," Acta Microbiologica et Immunologica Hungarica, vol. 60, no. 2, pp. 201-209, 2013.

[9] J. Le, B. McKee, W. Srisupha-Olarn, and D. S. Burgess, "In vitro activity of carbapenems alone and in combination with amikacin against KPC-producing Klebsiella pneumoniae," Journal of Clinical Medicine Research, vol. 3, pp. 101-110, 2011.

[10] S. Pournaras, G. Vrioni, E. Neou et al., "Activity of tigecycline alone and in combination with colistin and meropenem against
Klebsiella pneumoniae carbapenemase (KPC)-producing Enterobacteriaceae strains by time-kill assay," International Journal of Antimicrobial Agents, vol. 37, no. 3, pp. 244-247, 2011.

[11] C. Urban, N. Mariano, and J. J. Rahal, "In vitro double and triple bactericidal activities of doripenem, polymyxin B, and rifampin against multidrug-resistant Acinetobacter baumannii, Pseudomonas aeruginosa, Klebsiella pneumoniae, and Escherichia coli," Antimicrobial Agents and Chemotherapy, vol. 54, no. 6, pp. 2732-2734, 2010.

[12] P.-Y. Lee, W.-N. Chang, C.-H. Lu et al., "Clinical features and in vitro antimicrobial susceptibilities of community-acquired Klebsiella pneumoniae meningitis in Taiwan," Journal of Antimicrobial Chemotherapy, vol. 51, no. 4, pp. 957-962, 2003.

[13] G. Patel, S. Huprikar, S. H. Factor, S. G. Jenkins, and D. P. Calfee, "Outcomes of carbapenem-resistant Klebsiella pneumoniae infection and the impact of antimicrobial and adjunctive therapies," Infection Control and Hospital Epidemiology, vol. 29, no. 12, pp. 1099-1106, 2008.

[14] R. W. Bundtzen, A. U. Gerber, D. L. Cohn, and W. A. Craig, "Postantibiotic suppression of bacterial growth," Reviews of Infectious Diseases, vol. 3, no. 1, pp. 28-37, 1981.

[15] W. A. Craig and S. Gudmundsson, "The postantibiotic effect," in Antibiotics in Laboratory Medicine, V. Lorian, Ed., pp. 296-329, Williams and Wilkins, Baltimore, Md, USA, 4th edition, 1996.

[16] P. J. MacDonald, W. A. Craig, and C. M. Kunin, "Persistent effect of antibiotics on Staphylococcus aureus after exposure for limited periods of time," Journal of Infectious Diseases, vol. 135, no. 2, pp. 217-223, 1977.

[17] J. M. Spivey, "The postantibiotic effect," Clinical Pharmacy, vol. 11, no. 10, pp. 865-875, 1992.

[18] W. A. Craig, "The role of pharmacodynamics in effective treatment of community-acquired pathogens," Advanced Studies in Medicine, vol. 2, pp. 126-134, 2002.

[19] Clinical and Laboratory Standards Institute (CLSI), Methods for Dilution Antimicrobial Susceptibility Tests for Bacteria that Grow Aerobically, Approved Standard M7-A7, CLSI, Wayne, Ill, USA, 7th edition, 2006.

[20] R. Hope, M. Warner, S. Mushtaq, M. E. Ward, T. Parsons, and D. M. Livermore, "Effect of medium type, age and aeration on the MICs of tigecycline and classical tetracyclines," Journal of Antimicrobial Chemotherapy, vol. 56, no. 6, pp. 1042-1046, 2005.

[21] S. K. Pillai, R. C. Moellering Jr., and G. M. Eliopoulos, "Antimicrobial combinations," in Antibiotics in Laboratory Medicine, V. Lorian, Ed., pp. 365-440, Lippincott Williams and Wilkins, Philadelphia, Pa, USA, 2005.

[22] F. C. Odds, "Synergy, antagonism, and what the chequerboard puts between them," Journal of Antimicrobial Chemotherapy, vol. 52, no. 1, p. 1, 2003.

[23] National Committee for Clinical Laboratory Standards, Methods for Determining A Bactericidal Activity of Antimicrobial Agents: Approved Guideline M26-A, National Committee for Clinical Laboratory Standards, Wayne, Pa, USA, 1999.

[24] S. K. Spangler, S. Bajaksouzian, M. R. Jacobs, and P. C. Appelbaum, "Postantibiotic effects of grepafloxacin compared to those of five other agents against 12 gram-positive andnegative bacteria," Antimicrobial Agents and Chemotherapy, vol. 44, no. 1, pp. 186-189, 2000.

[25] B. Özbek and A. Şentürk, "Postantibiotic effects of tigecycline, colistin sulfate, and levofloxacin alone or tigecycline-colistin sulfate and tigecycline-levofloxacin combinations against Acinetobacter baumannii," Chemotherapy, vol. 56, no. 6, pp. 466$471,2010$. 
[26] Ç. Bozkurt-Güzel and A. A. Gerçeker, "Post-antibiotic effect of colistin, alone and in combination with amikacin, on Pseudomonas aeruginosa strains isolated from cystic fibrosis patients," Journal of Antibiotics, vol. 65, no. 2, pp. 83-86, 2012.

[27] J. Li, J. Turnidge, R. Milne, R. L. Nation, and K. Coulthard, "In vitro pharmacodynamic properties of colistin and colistin methanesulfonate against Pseudomonas aeruginosa isolates from patients with cystic fibrosis," Antimicrobial Agents and Chemotherapy, vol. 45, no. 3, pp. 781-785, 2001.

[28] R. J. Owen, J. Li, R. L. Nation, and D. Spelman, "In vitro pharmacodynamics of colistin against Acinetobacter baumannii clinical isolates," Journal of Antimicrobial Chemotherapy, vol. 59, no. 3, pp. 473-477, 2007.

[29] B. Ozbek and G. Otuk, "Post-antibiotic effect of levofloxacin and tobramycin alone or in combination with cefepime against Pseudomonas aeruginosa," Chemotherapy, vol. 55, no. 6, pp. 446-450, 2009.

[30] S. Gudmundsson, S. Einarsson, H. Erlendsdottir, J. Moffat, W. Bayer, and W. A. Craig, "The post-antibiotic effect of antimicrobial combinations in a neutropenic murine thigh infection model," Journal of Antimicrobial Chemotherapy, vol. 31, pp. 177-191, 1993.

[31] G. A. Pankuch and P. C. Appelbaum, "Postantibiotic effect of tigecycline against 14 gram-positive organisms," Antimicrobial Agents and Chemotherapy, vol. 53, no. 2, pp. 782-784, 2009.

[32] A. Lefort, M. Lafaurie, L. Massias et al., "Activity and diffusion of tigecycline (GAR-936) in experimental enterococcal endocarditis," Antimicrobial Agents and Chemotherapy, vol. 47, no. 1, pp. 216-222, 2003.

[33] W. Stubbings, J. Bostock, E. Ingham, and I. Chopra, "Mechanisms of the post-antibiotic effects induced by rifampicin and gentamicin in Escherichia coli," Journal of Antimicrobial Chemotherapy, vol. 58, no. 2, pp. 444-448, 2006.

[34] J. Dubois and C. St-Pierre, "Comparative in vitro activity and post-antibiotic effect of gemifloxacin against Legionella spp," Journal of Antimicrobial Chemotherapy, vol. 45, no. 4, pp. 41-46, 2000.

[35] I. Odenholt-Tornqvist, "Studies on the postantibiotic effect and the postantibiotic sub-MIC effect of meropenem," Journal of Antimicrobial Chemotherapy, vol. 31, no. 6, pp. 881-892, 1993.

[36] D. N. Gilbert, "Once-daily aminoglycoside therapy," Antimicrobial Agents and Chemotherapy, vol. 35, no. 3, pp. 399-405, 1991.

[37] J. M. Prins, H. R. Buller, E. J. Kuijper, R. A. Tange, and P. Speelman, "Once versus thrice daily gentamicin in patients with serious infections," The Lancet, vol. 341, no. 8841, pp. 335-339, 1993.

[38] C. D. Freeman, D. P. Nicolau, P. P. Belliveau, and C. H. Nightingale, "Once-daily dosing of aminoglycosides: review and recommendations for clinical practice," Journal of Antimicrobial Chemotherapy, vol. 39, no. 6, pp. 677-686, 1997.

[39] D. S. Burgess, "Pharmacodynamic principles of antimicrobial therapy in the prevention of resistance," Chest, vol. 115, supplement 3, pp. 19-23, 1999.

[40] G. L. Drusano, D. E. Johnson, M. Rosen, and H. C. Standiford, "Pharmacodynamics of a fluoroquinolone antimicrobial agent in a neutropenic rat model of Pseudomonas sepsis," Antimicrobial Agents and Chemotherapy, vol. 37, no. 3, pp. 483-490, 1993.

[41] K. A. Rodvold and M. Neuhauser, "Pharmacokinetics and pharmacodynamics of fluoroquinolones," Pharmacotherapy, vol. 21, no. 10, pp. 233S-252S, 2001.
[42] P. Kardas, "Patient compliance with antibiotic treatment for respiratory tract infections," Journal of Antimicrobial Chemotherapy, vol. 49, no. 6, pp. 897-903, 2002.

[43] C. Tascini, E. Tagliaferri, T. Giani et al., "Synergistic activity of colistin plus rifampin against colistin-resistant kpc-producing Klebsiella pneumoniae," Antimicrobial Agents and Chemotherapy, vol. 57, no. 8, pp. 3990-3993, 2013.

[44] A. A. Gerceker and G. Otuk, "Postantibiotic effect of imipenem, alone and in combination with amikacin, on Pseudomonas aeruginosa," Chemotherapy, vol. 41, no. 6, pp. 433-436, 1995.

[45] I. M. Gould, A. C. Jason, and K. Milne, "Use of the malthus microbial growth analyser to study the post antibiotic effect of antibiotics," Journal of Antimicrobial Chemotherapy, vol. 24, no. 4, pp. 523-531, 1989. 

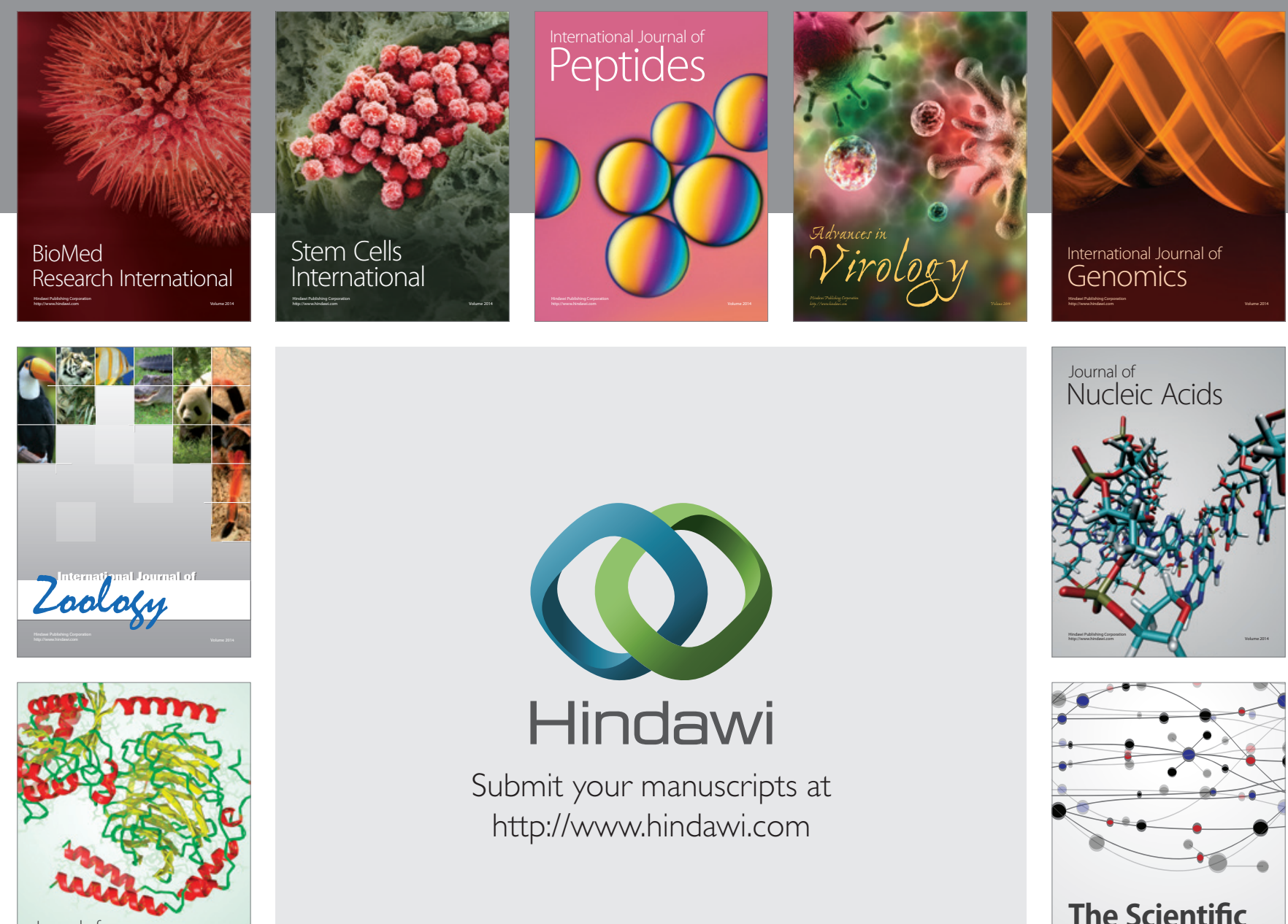

Submit your manuscripts at

http://www.hindawi.com

Journal of
Signal Transduction
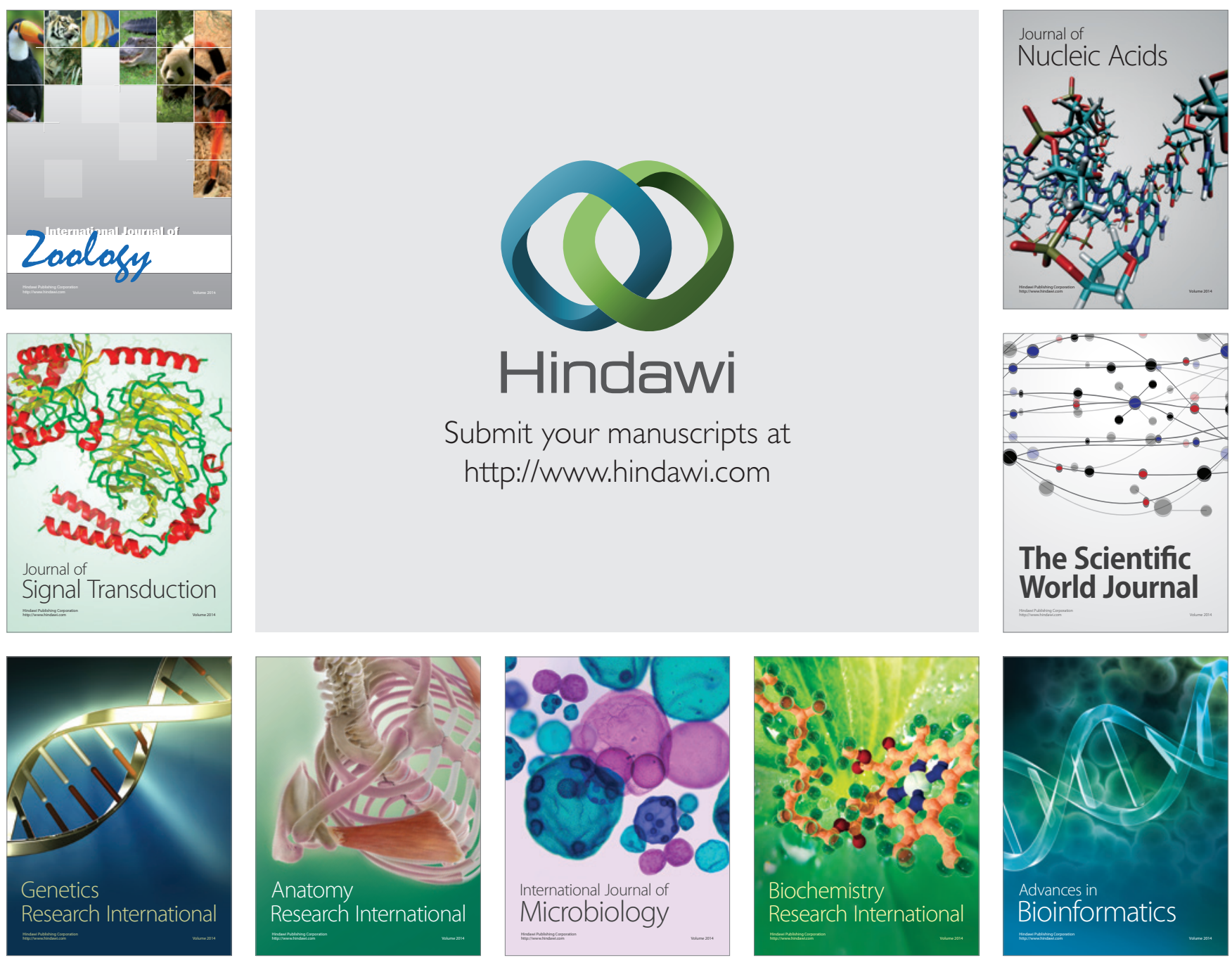

The Scientific World Journal
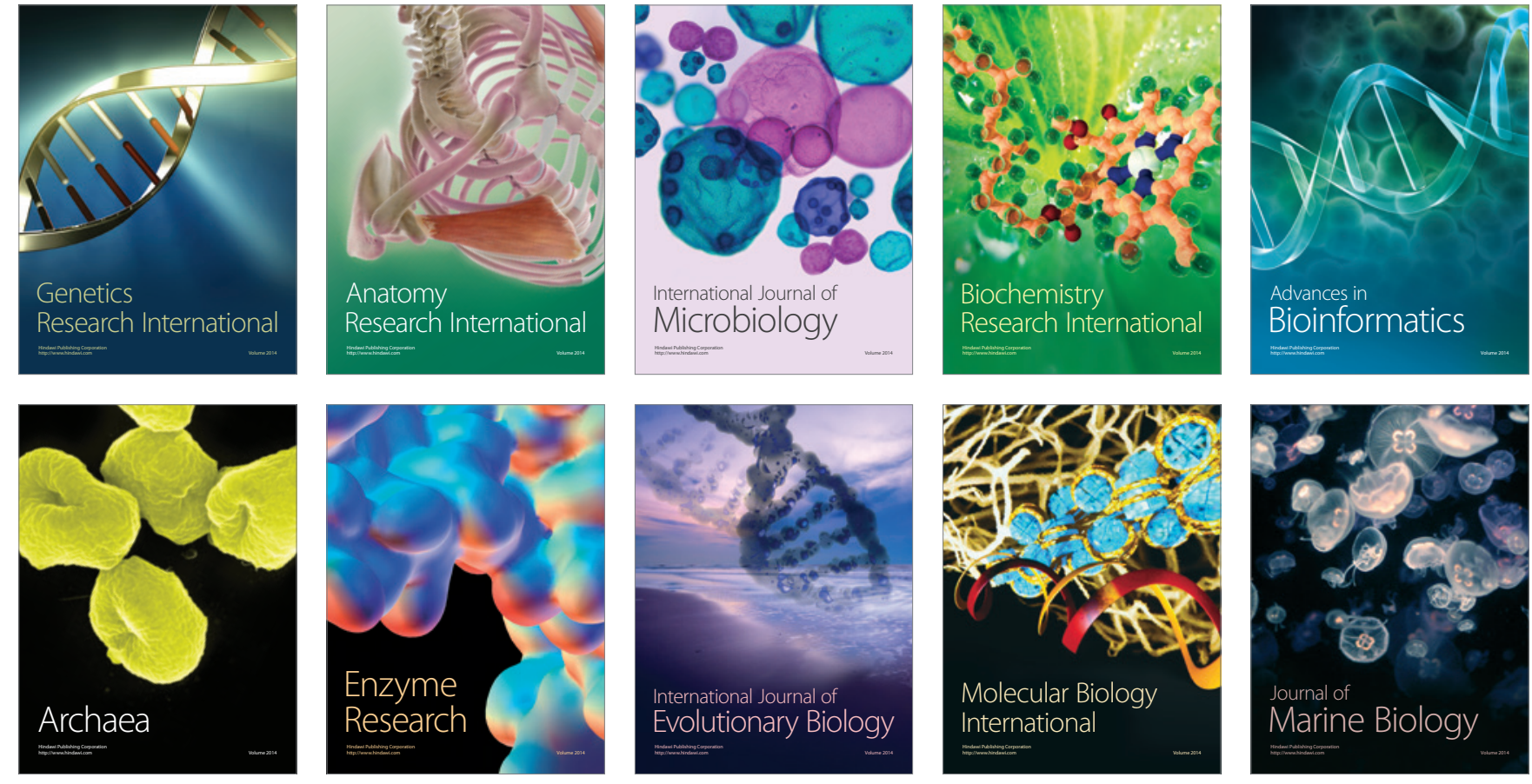